\title{
Normobaric hyperoxia-based neuroprotective therapies in ischemic stroke
}

\author{
Zhifeng $\mathrm{Qi}^{1}$, Wenlan Liü ${ }^{2,3}$, Yumin Luo ${ }^{1}$, Xunming Ji and Ke Jian Liư ${ }^{1,3^{*}}$
}

\begin{abstract}
Stroke is a leading cause of death and disability due to disturbance of blood supply to the brain. As brain is highly sensitive to hypoxia, insufficient oxygen supply is a critical event contributing to ischemic brain injury. Normobaric hyperoxia (NBO) that aims to enhance oxygen delivery to hypoxic tissues has long been considered as a logical neuroprotective therapy for ischemic stroke. To date, many possible mechanisms have been reported to elucidate NBO's neuroprotection, such as improving tissue oxygenation, increasing cerebral blood flow, reducing oxidative stress and protecting the blood brain barrier. As ischemic stroke triggers a battery of damaging events, combining NBO with other agents or treatments that target multiple mechanisms of injury may achieve better outcome than individual treatment alone. More importantly, time loss is brain loss in acute cerebral ischemia. NBO can be a rapid therapy to attenuate or slow down the evolution of ischemic tissues towards necrosis and therefore "buy time" for reperfusion therapies. This article summarizes the current literatures on NBO as a simple, widely accessible, and potentially cost-effective therapeutic strategy for treatment of acute ischemic stroke.
\end{abstract}

Keywords: Oxygen, Ischemia, Oxidative stress, Blood brain barrier, Reperfusion, Blood flow, Neuroprotection

\section{Introduction}

Stroke is a leading cause of death and disability. Acute ischemic stroke results in heterogeneous changes in cerebral blood flow (CBF) and brain metabolism in the affected region [1]. As brain is extremely sensitive to hypoxia, insufficient oxygen supply due to disturbance in the cerebral blood supply is a critical event in the pathophysiology of ischemic stroke. Therefore, oxygen therapy that aims at improving tissue oxygen supply has long been considered as a logical treatment for ischemic stroke [2]. Recent animal studies have shown that short duration of normobaric hyperoxia (NBO) treatment is highly neuroprotective if started early after stroke onset [3-5]. As time loss is brain loss in the setting of acute cerebral ischemia, NBO can be administered as an early treatment to attenuate or slow down the evolution of ischemic tissue towards necrosis, and thus "buy time" for combination therapies with other neuroprotectants to achieve better outcomes [6,7]. In this review, we will

\footnotetext{
* Correspondence: kliu@salud.unm.edu

${ }^{1}$ Cerebrovascular Diseases Research Institute, Xuanwu Hospital of Capital Medical University, No.45 Changchun Street, Beijing 100053, China

${ }^{3}$ Department of Pharmaceutical Sciences, University of New Mexico Health

Sciences Center, Albuquerque, NM 87131, USA

Full list of author information is available at the end of the article
}

summarize the current literatures on the neuroprotective studies of NBO alone and its combination with other therapies in acute ischemic stroke.

\section{NBO-afforded neuroprotection in stroke}

\section{NBO: an ideal therapy for acute ischemic stroke}

Oxygen, a commonly used therapeutic agent, has several properties that make it an "ideal" neuroprotective drug $[8,9]$. Firstly, as a small hydrophobic molecule, oxygen can easily diffuse across the blood brain barrier (BBB) to improve oxygen supply to brain tissue [10]. Secondly, short durations of oxygen are relatively safe, well tolerated, and without dose-limiting side-effects except in acute flare patients with chronic obstructive pulmonary diseases [11]. Thirdly, NBO is widely available and commonly prescribed by medical staff in a broad range of conditions to relieve or prevent tissue hypoxia. NBO can be simply administered by paramedics or even at home, which makes it an ideal acute stroke therapy. Increasing evidence from animal stroke studies has shown that NBO can reduce ischemic brain injury and improve functional outcome [12-18]. Moreover, human studies also demonstrate that NBO is associated with a transient improvement of clinical deficits and magnetic resonance

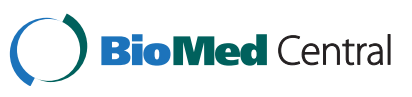


imaging (MRI) abnormalities in patients with acute ischemic stroke [19-21]. The neuroprotection of NBO are explained by many possible mechanisms, such as improving metabolism, increasing $\mathrm{CBF}$, reducing oxidative stress, and protecting microvasculature.

\section{NBO improves tissue oxygenation and metabolism in the ischemic brain}

Hypoxia is a critical event leading to neuronal death in stroke. Acute ischemic stroke results in heterogeneous changes in tissue oxygenation, with a moderately reduced blood flow in the penumbra and a severely hypoperfused core [1]. Using electron paramagnetic resonance oximetry to measure localized interstitial partial oxygen $\left(\mathrm{pO}_{2}\right)$ in a rat model, Liu et al. reported that interstitial $\mathrm{pO}_{2}$ rapidly decreased to about $30 \%$ of pre-ischemic values in penumbra and $4 \%$ in the core during cerebral ischemia [22]. They further showed that 95\% normobaric oxygen given during ischemia was able to maintain penumbral interstitial $\mathrm{pO}_{2}$ levels close to the pre-ischemic value while it caused a twofold increase in penumbral $\mathrm{pO}_{2}$ level if given during reperfusion [13].

However, whether NBO improves tissue oxygenation in stroke patients is still unkown. In light of studies on patients with traumatic brain injury that NBO increases brain tissue oxygen levels [23], primate or clinical studies are needed to explore brain tissue oxygenation in stroke during NBO therapy.

Abnormal metabolism is another major cause of brain injury under ischemic/hypoxic condition. NBO has been shown to reduce tissue acidosis and ATP depletion in the border zones of focal cerebral ischemia [24]. Multivoxel magnetic resonance spectroscopic imaging from stroke patients with inhalation oxygen in a flow of $45 \mathrm{~L} /$ min through a face mask for 8 hours indicated that lactate (a marker for aerobic metabolism) decreased during $\mathrm{NBO}$ administration, suggesting that NBO improves aerobic metabolism in stroke patients [21]. As the penumbra is the region that can potentially be rescued if blood flow is restored in time [25], NBO may work as an early intervention to preserve the penumbra and expand the time window of reperfusion therapies.

Inspired by recent reports on postconditioning with intermittent occlusions of cerebral artery, Liu et al. recently showed that intermittent NBO treatment (4 short cycles of intermittent NBO/air treatment) provided similar neuroprotection at 24 hours after ischemia onset and even greater neuroprotection at 72 hours, when compared to continuous NBO [26]. Their results suggest that besides providing oxygen to the ischemic tissue, intermittent NBO must have exerted its neuroprotection through other mechanisms. It is conceivable that intermittent NBO may trigger a novel form of postconditioning, in which the observed neuroprotection may be mediated by attenuating superoxide generation and activation of the Akt pathway.

These findings suggest that, in addition to improving oxygen supply and metabolism, NBO could induce endogenous protective mechanisms to reduce ischemic brain injury.

\section{Changes in cerebral blood flow}

It is well accepted that healthy tissue responds to hyperoxia with consistent vasoconstriction [27]. How the CBF changes in ischemic tissue in response to NBO therapy is an important question. An early study from Liu et al. showed that NBO given during cerebral ischemia increased $\mathrm{CBF}$ in the ischemic penumbra, which was accompanied by a reduction in $\mathrm{CBF}$ in the contralateral hemisphere [13]. When NBO was delivered during reperfusion, it significantly decreased penumbral CBF. The blood flow in the core region was not affected by NBO during both ischemia and reperfusion [13]. Using real-time two-dimensional multispectral reflectance imaging and laser speckle flowmetry, Shin et al. reported that NBO significantly increased the CBF in ischemic cortex and reduced the cortical infarction in comparison to room air 1 hour after middle cerebral artery occlusion (MCAO) onset [28]. A study using functional cerebral blood volume (fCBV) also showed that contralateral healthy tissue responded consistently with vasoconstriction (fCBV reduction), while tissue from ischemic core showed marginal fCBV changes that later became moderate fCBV reductions. As for penumbra tissue, it showed relative preservation of mean $\mathrm{ACBV}$ at early time points, later exhibited significantly decreased fCBV (vasoconstriction) like healthy tissue [16].

The study from Henninger et al. indicated that NBO acutely preserved the perfusion/diffusion mismatch without altering $\mathrm{CBF}$, because $\mathrm{CBF}$ characteristics and CBF-derived lesion volumes did not differ between NBO-treated and untreated animals [12]. The authors explained that the observed mild elevation of $\mathrm{PaCO}_{2}$ may have counterbalanced the vasoconstrictive effects associated with hyperoxia, suggesting that CBF changes were unlikely to be a major mechanism of neuroprotection in the acute phase of ischemia in this model.

Taken together, change of CBF in ischemic tissue in response to NBO is complicated, and whether CBF augmentation would eventually benefit stroke animals or patients needs to be investigated further.

\section{Impact on oxidative stress}

Reactive oxygen species (ROS) may be generated when tissue oxygen level is too high or too low [29]. Oxidative stress has been widely considered as one of important mechanisms in ischemic and/or reperfusion injury [30,31]. Evidence from recent animal studies has demonstrated that short duration of $\mathrm{NBO}$ treatment does not increase oxidative 
stress if started early after stroke onset. Agardh et al. indicated that recirculation following brief periods of ischemia (15 min) did not lead to an enhanced $\mathrm{H}_{2} \mathrm{O}_{2}$ production with the aminotriazole/catalase method, and that hyperoxia did not aggravate the ischemic damage [32]. Liu et al. have shown that NBO given during cerebral ischemia reduces superoxide production and the generation of 8 -OHdG, a biomarker of oxidative DNA damage [13]. In an attempt to identify the underlying mechanism, Liu et al. found that NBO inhibited the upregulation of gp91 ${ }^{\text {phox }}$, the catalytic subunit of NADPH oxidase [33]. In addition, when gp91 ${ }^{\text {phox }}$ was knocked out in the transgenic mouse model, $\mathrm{NBO}$ was no longer able to induce reduction in BBB leakage [14]. These results are further supported by the report that NBO treatment during focal cerebral ischemiareperfusion does not increase oxidative stress, as measured by heme oxygenase- 1 induction and protein carbonyl formation [34]. Findings from these studies indicate that NBO treatment decreases ROS production in the penumbra when penumbral $\mathrm{pO}_{2}$ is maintained close to the preischemic level.

The effect of $\mathrm{NBO}$ on reactive nitrogen species (RNS) generation has also been examined in an animal stroke model. Yuan et al. recently showed NBO given during cerebral ischemia delayed and attenuated early nitric oxide generation, probably through inhibiting neuronal nitric oxide synthase (nNOS) [35]. In this study, they found that ischemia caused rapid production of $\mathrm{NO}_{x}^{-}$(nitrite plus nitrate), with a peak at $10 \mathrm{~min}$ after stroke onset, then gradually declining to the baseline level at $60 \mathrm{~min}$. NBO delayed the $\mathrm{NO}_{x}^{-}$peak to $30 \mathrm{~min}$ and attenuated the total amount of $\mathrm{NO}_{x}^{-}$. Moreover, NBO showed similar inhibitory effect on $\mathrm{NO}_{x}^{-}$and 3-nitrotyrosine production as that of the specific nNOS inhibitor 7-nitroindazole.

Together, these studies suggest that, contrary to common belief, under appropriate conditions NBO treatment would not cause observable increase of oxidative stress, while it may actually decrease oxidative stress in the penumbra. However, the underlying mechanism is not fully understood, and will require further investigation.

The findings from the studies on oxidative stress also suggest that the brain is more tolerable to hyperoxia than hypoxia. Although $\mathrm{NBO}$ treatment elevates tissue $\mathrm{pO}_{2}$ in the contralateral brain to a level that is much higher than the physiologic value, it did not increase ROS production [13]. Furthermore, when 95\% oxygen was delivered during reperfusion, penumbral $\mathrm{pO}_{2}$ level was twice of the preischemic level, however no significant increase in ROS generation was observed [13]. These findings may be explained by different CBF responses to NBO in healthy, penumbral and core tissues [16]. Vasoconstriction is the normal function for healthy vasculature to avoid oxygen overload and the risk of oxidative stress. As for ischemic tissue, loss of functional vasoconstriction (vasodilation) in response to hyperoxia fortunately increases CBV and oxygen supply to overcome hypoxia. Once the tissue is rescued, the functional vasoconstriction may also return to avoid excessive oxygen supply.

\section{Protection on blood brain barrier}

Blood brain barrier (BBB) is a physical and metabolic diffusion barrier between cerebral microvessels and the surrounding tissue, which is essential for the maintenance of homeostasis and the normal function of the central nervous system [2,36]. Matrix metalloproteinases (MMPs) have been the focus of many studies of cerebral ischemia because of their substrate specificity for fibronectin, laminin, collagen type IV and tight junction proteins (TJPs), which are structural components of the BBB [37].

In stroke animal models, the activity or expression of MMP-2 and -9 is significantly increased, which is closely related to $\mathrm{BBB}$ disruption, edema formation and intracranial hemorrhage [38-45]. Evidence from animal stroke studies shows that NBO inhibits MMP-9 induction in the ischemic brain, leading to reduction in occludin degradation, Evan's blue extravasation and hemispheric swelling [3,13,33]. The mechanism by which NBO suppresses MMP-9 and attenuates $\mathrm{BBB}$ damage appears to involve NADPH oxidase, because i) inhibition of NADPH oxidase with apocynin or knockout of gp91 ${ }^{\text {phox }}$ resulted in much smaller magnitudes in MMP-9 induction and BBB leakage in the ischemic brain $[13,14,33]$, and ii) NBO did not cause additional reduction in MMP-9 induction when gp91 ${ }^{\text {phox }}$ was knocked out [14]. These results indicate that inhibition of NADPH oxidasederived ROS production may be an important mechanism underlying NBO-afforded $\mathrm{BBB}$ protection. As the tightness of the $\mathrm{BBB}$ is crucial to ensure the ischemic brain to safely withstand the return of blood flow, early treatment with NBO may "buy time" for thrombolytic therapy by attenuating or slowing down the disruption of the BBB.

\section{Controversies in NBO protection}

Although a large pool of evidence has demonstrated that $\mathrm{NBO}$ is beneficial if it is given during cerebral ischemia, there are also controversial reports. For example, Li et al. reported that they failed to see a significant decrease in the infarct size [46]. In another study, NBO therapy for a 24 hour period was shown to reduce brain swelling in MCAO rats, but had no effect on brain infarction [46]. Perhaps, NBO for extended time (e.g., 24 hour) might reduce its neuroprotective effect. In an attempt to explore the long-term effect of NBO on ischemic brain injury, Mickel et al. showed that NBO resulted in a more severe damage to myelin sheaths in gerbils that survived 28 days after stroke onset, although neurons in the deeper laminae of the cerebral cortex appeared to be better preserved in these animals [47]. 
Different results have also been reported in human stroke studies regarding to NBO's neuroprotection. Singhal et al. reported that NBO therapy via facemask for 8 hours improves stroke scale scores at 24 hours in NBO-treated patients, but not at 3 months [20]. In addition, mean relative diffusion MRI lesion volumes were also significantly reduced in NBO-treated patients at 4 hours but not subsequent time points. These findings indicated that NBO's effects might be transient. Another study by Chiu et al. showed that breathing 40\% oxygen using venturi mask decreased mortality and comorbidities in patients experiencing a first-ever large MCA infarction (more than one third of the MCA territory on brain images) within 48 hours [19]. On the contrary, Padma et al. reported that $\mathrm{NBO}$ in a flow of $2 \mathrm{~L} / \mathrm{min}$ failed to improve the clinical scores of mean National Institute of Health stroke scale (NIHSS), modified Rankin score, Barthel index at 0, 1, 7 day of admission and at 3 months follow-up in stroke patients [48]. Obviously, better-designed clinical studies with well defined patient groups (e.g., the optimum concentration of oxygen inhalation and time after stroke onset) are needed to further investigate the safety and efficacy of NBO as a stroke therapy.

\section{Combination treatment with NBO}

The Stroke Therapy Academic Industry Roundtable (STAIR) has strongly recommended the use of multiple neuroprotective therapies for treating stroke, in which each agent that works on a different ischemic injury mechanism could be given either simultaneously or in rapid succession $[49,50]$. As NBO is widely available and can be promptly initiated after stroke onset, NBO may be an ideal early stroke treatment to preserve the ischemic penumbra, and then followed by treatment with other neuroprotectants to eventually salvage the ischemic penumbral tissue.

\section{NBO plus tPA thrombolysis}

A major goal in the treatment of acute ischemic stroke is prompt arterial recanalization. Until now, thrombolysis with tissue-type plasminogen activator (tPA) within 3 or 4.5 hour after symptom onset is the only FDAapproved treatment for acute ischemic stroke. Delayed thrombolytic therapy dramatically increases the risk of intracranial hemorrhage ( $\mathrm{ICH})$ [51]. As a fact, the brief therapeutic window and the high incidence of $\mathrm{ICH}$ have profoundly constrained the clinical use of tPA in ischemic stroke patients. Therefore, combination strategies that address not only tPA-associated $\mathrm{ICH}$ and its narrow therapeutic window, but also limit ischemic damage [49], are urgently needed.

Although the mechanisms underlying tPA-induced $\mathrm{ICH}$ are not fully understood, it has been suggested that $\mathrm{ICH}$ occurs as a result of BBB disruption by enhancing the proteolytic activity, such as MMPs [52-56]. Several human studies have also demonstrated that stroke patients with higher pretreatment plasma level of MMP9 or its substrate are more likely to undergo cerebral hemorrhagic complications after tPA $[57,58]$.

Since NBO can effectively limit ischemic neuronal death, as well as reduce $\mathrm{BBB}$ disruption in animal experiments $[14,34]$, it is logic to speculate that NBO could serve as a promising adjunctive therapy to reduce tPA-associated $\mathrm{ICH}$ and widen the thrombolysis window in stroke. The potential for combined NBO treatment with tPA thrombolysis was recognized several years ago [15,59]. Henninger et al. showed that the combination of NBO with tPA did not increase hemorrhage volume at 10 hours or occurrence of confluent petechial

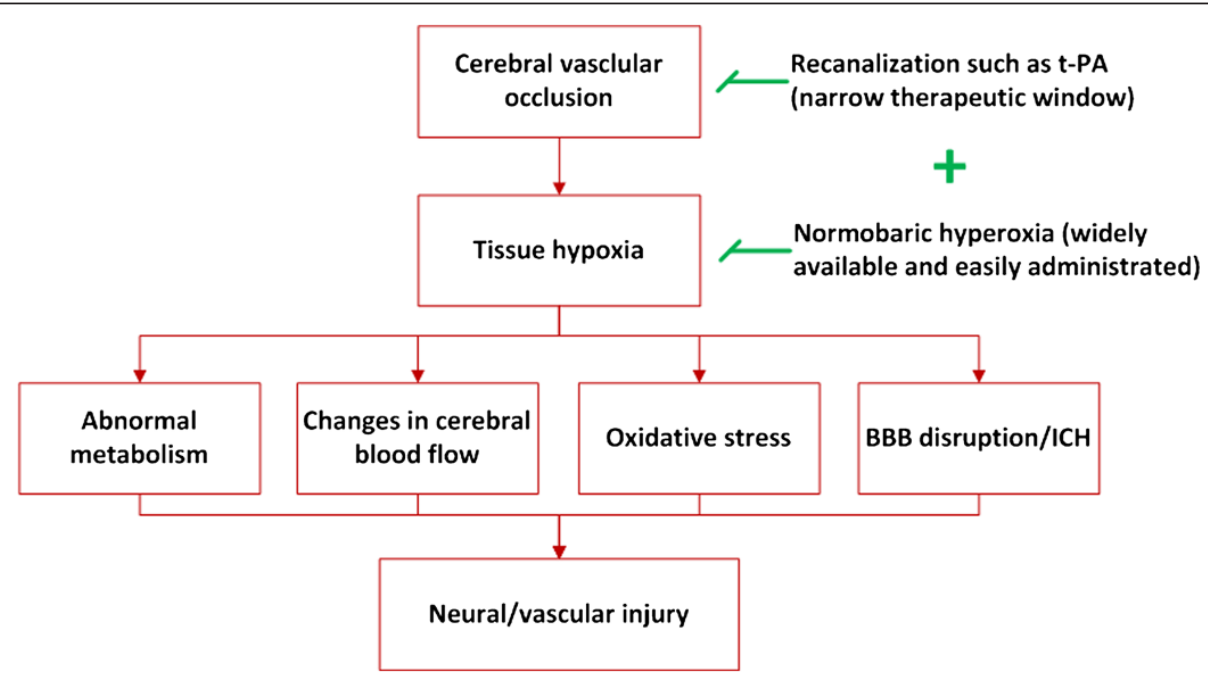

Figure 1 The schematic diagram of neuroprotection of NBO alone or combination therapy in ischemic stroke. 
hemorrhages at 24 hours in a rat embolic stroke model [60]. NBO also did not increase cellular markers of superoxide generation or brain levels of MMP-9 [61]. These data provides important initial evidence to support the combination therapy with NBO and tPA in ischemic stroke.

It was reported that combined $\mathrm{NBO}$ and tPA significantly reduced the mortality rate (from $70 \%$ to $11 \%$ in tPA-treated rats), brain edema, hemorrhage, and MMP-9 augmentation in a filament occlusion rat model with 5hour ischemia followed by 19 -hour reperfusion, as compared with tPA alone [6]. The authors also pointed out that they could not draw a solid conclusion on the causal relationship between mortality and cerebral hemorrhage, as they did not measure hemorrhage volume for those rats that died prematurely during reperfusion, most of which were tPA-treated normoxic rats. Nevertheless, their findings indicate that NBO can increase the safety of delayed tPA treatment. However, different results have also been reported, in which Fujiwara et al. showed that NBO plus tPA did not significantly alter hemorrhagic conversion, brain swelling, or mortality at 24 hours, as compared with tPA alone in an embolic MCAO model stroke [62]. The discrepancy might be derived from the difference in stability of animal models. Apparently, further investigation is needed to ascertain the safety and efficacy of delayed tPA treatment combined with NBO before application in human stroke patients.

\section{NBO plus other therapies}

Minocycline, a tetracycline antibiotic, possesses properties against reperfusion injury, including anti-inflammatory, anti-apoptotic, and BBB protection. Jin et al. reported that NBO therapy after ischemia onset plus minocycline intravenous injection after reperfusion onset provides greater reduction in infarct size and edema volume. NBO plus minocycline also effectively reduces brain injury due to its inhibition on MMP-2/9-mediated occludin degradation and the activation of caspases and apoptosis inducing factor [63].

Effects of combination treatment with NBO and edaravone, a potent scavenger of hydroxyl radicals (intravenously injected after ischemia), were evaluated for reducing acute brain injury in a 2-hour filament ischemic mouse model [64]. This study indicated that combination group showed smaller infarct volume, better neurological functions and less TUNEL-positive cells in the ischemic boundary zone both in cortex and subcortex at 22 hours after reperfusion than the two monotherapy groups, suggesting that combination therapy with NBO plus edaravone prevented the neuronal damage after focal cerebral ischemia and reperfusion in mice. Combination therapy with NBO plus cilostazol, a selective inhibitor of phosphodiesterase-3, was also proved to protect the mice subjected to focal cerebral ischemia by improving CBF after reperfusion, which was partially attributed to endothelial nitric-oxide synthase activity [65].

NBO has also been suggested to combine with ultrasound and tPA in acute ischemic stroke [66]. Enhanced dissolved oxygen secondary to NBO treatment increases gas nuclei formation around and inside of the clot. Under ultrasound field, these small gas nuclei form nano bubbles that fuel inertial cavitation as substrates, and therefore increase the clot fragmentation and lysis.

Since multiple mechanisms contribute to ischemic injury, it is logic and appropriate to consider the "cocktail" approach to intervene multiple mechanisms. Moreover, combined therapies are also helpful for reducing the dosage and minimizing the toxicity of individual agent.

\section{Conclusions}

At present, stroke patients receive variable amounts of oxygen in the ambulance and current guidelines do not support the routine use of in-hospital oxygen $[67,68]$. In light of the positive preclinical and clinical reports, further studies are warranted to explore the efficacy of NBO therapy alone or in combination with other neuroprotectants for treatment of acute ischemic stroke, and to determine the optimum timing and duration of the therapy. NBO, a simple, widely accessible and potentially cost-effective therapeutic strategy could significantly improve the clinical outcome of stroke patients if used appropriately (Figure 1).

\section{Abbreviations}

BBB: Blood brain barrier; CBF: Cerebral blood flow; fCBV: Functional cerebral blood volume; ICH: Intracranial hemorrhage; MCAO: Middle cerebral artery occlusion; MMPs: Matrix metalloproteinases; MRI: Magnetic Resonance Imaging; NBO: Normobaric hyperoxia; NIHSS: National Institute of Health stroke scale; nNOS: Neuronal nitric oxide synthase; NOx-: Nitrite plus nitrate; $\mathrm{pO}_{2}$ : Partial oxygen; ROS: Reactive nitrogen species; RNS: Reactive nitrogen species; STAIR: Stroke Therapy Academic Industry Roundtable; TJPs: Tight junction proteins; tPA: Tissue-type plasminogen activator.

\section{Competing interests}

The authors declare that they have no competing interests.

\section{Authors' contributions}

ZFQ reviewed the NBO studies in stroke, wrote up the manuscript and obtained partial funding. WLL edited the overall manuscript and improved the structure of this review. YML and XMJ reviewed the NBO studies in clinic. $K J \mathrm{~L}$ participated in the overall design of this review and obtained partial funding. All authors have read and approved the final manuscript.

\section{Acknowledgements}

The work was partially supported by grants from National Natural Science Foundation of China (81171242 and 81200928), and National Institutes of Health, USA (P30GM103400, and R01AG031725).

\section{Author details}

${ }^{1}$ Cerebrovascular Diseases Research Institute, Xuanwu Hospital of Capital Medical University, No.45 Changchun Street, Beijing 100053, China. ${ }^{2}$ Central Laboratory of Shenzhen 2nd People's Hospital, the 1st Affiliated Hospital of 
Shenzhen University, 3002 Sungang West Rd, Shenzhen, Guangdong 518035, China. ${ }^{3}$ Department of Pharmaceutical Sciences, University of New Mexico Health Sciences Center, Albuquerque, NM 87131, USA.

Received: 23 November 2012 Accepted: 7 January 2013

Published: 9 January 2013

\section{References}

1. Eltzschig HK, Eckle T: Ischemia and reperfusion-from mechanism to translation. Nat Med 2011, 17(11):1391-1401.

2. Rosenberg GA: Neurological diseases in relation to the blood-brain barrier. J Cereb Blood Flow Metab 2012, 32(7):1139-1151.

3. Liu W, Hendren J, Qin XJ, Shen J, Liu KJ: Normobaric hyperoxia attenuates early blood-brain barrier disruption by inhibiting MMP-9-mediated occludin degradation in focal cerebral ischemia. J Neurochem 2009, 108(3):811-820.

4. Michalski D, Hartig W, Schneider D, Hobohm C: Use of normobaric and hyperbaric oxygen in acute focal cerebral ischemia - a preclinical and clinical review. Acta Neurol Scand 2011, 123(2):85-97.

5. Singhal AB: A review of oxygen therapy in ischemic stroke. Neurol Res 2007, 29(2):173-183.

6. Liu W, Hendren J, Qin XJ, Liu KJ: Normobaric hyperoxia reduces the neurovascular complications associated with delayed tissue plasminogen activator treatment in a rat model of focal cerebral ischemia. Stroke 2009, 40(7):2526-2531.

7. Singhal AB: Oxygen therapy in stroke: past, present, and future. Int $J$ Stroke 2006, 1(4):191-200.

8. Liu W, Khatibi N, Sridharan A, Zhang JH: Application of medical gases in the field of neurobiology. Med Gas Res 2011, 1(1):13

9. Poli S, Veltkamp R: Oxygen therapy in acute ischemic stroke - experimental efficacy and molecular mechanisms. Curr Mol Med 2009, 9(2):227-241.

10. Hawkins BT, Egleton RD: Pathophysiology of the blood-brain barrier: animal models and methods. Curr Top Dev Biol 2008, 80:277-309.

11. Singhal AB, Lo EH, Dalkara T, Moskowitz MA: Advances in stroke neuroprotection: hyperoxia and beyond. Neuroimaging Clin N Am 2005, 15(3):697-xiii.

12. Henninger N, Bouley J, Nelligan JM, Sicard KM, Fisher M: Normobaric hyperoxia delays perfusion/diffusion mismatch evolution, reduces infarct volume, and differentially affects neuronal cell death pathways after suture middle cerebral artery occlusion in rats. J Cereb Blood Flow Metab 2007, 27(9):1632-1642

13. Liu S, Liu W, Ding W, Miyake M, Rosenberg GA, Liu KJ: Electron paramagnetic resonance-guided normobaric hyperoxia treatment protects the brain by maintaining penumbral oxygenation in a rat model of transient focal cerebral ischemia. J Cereb Blood Flow Metab 2006, 26(10):1274-1284.

14. Liu W, Chen Q, Liu J, Liu KJ: Normobaric hyperoxia protects the blood brain barrier through inhibiting Nox2 containing NADPH oxidase in ischemic stroke. Med Gas Res 2011, 1(1):22.

15. Singhal AB, Dijkhuizen RM, Rosen BR, Lo EH: Normobaric hyperoxia reduces MRI diffusion abnormalities and infarct size in experimental stroke. Neurology 2002, 58(6):945-952.

16. Wu O, Lu J, Mandeville JB, Murata Y, Egi Y, Dai G, et al: Dynamic functional cerebral blood volume responses to normobaric hyperoxia in acute ischemic stroke. J Cereb Blood Flow Metab 2012, 32(9):1800-1809.

17. Flynn EP, Auer RN: Eubaric hyperoxemia and experimental cerebral infarction. Ann Neurol 2002, 52(5):566-572.

18. Miyamoto O, Auer RN: Hypoxia, hyperoxia, ischemia, and brain necrosis. Neurology 2000, 54(2):362-371.

19. Chiu EH, Liu CS, Tan TY, Chang KC: Venturi mask adjuvant oxygen therapy in severe acute ischemic stroke. Arch Neurol 2006, 63(5):741-744.

20. Singhal AB, Benner T, Roccatagliata L, Koroshetz WJ, Schaefer PW, Lo EH, et al: A pilot study of normobaric oxygen therapy in acute ischemic stroke. Stroke 2005, 36(4):797-802.

21. Singhal AB, Ratai E, Benner T, Vangel M, Lee V, Koroshetz WJ, et al: Magnetic resonance spectroscopy study of oxygen therapy in ischemic stroke. Stroke 2007, 38(10):2851-2854

22. Liu S, Shi H, Liu W, Furuichi T, Timmins GS, Liu KJ: Interstitial pO2 in ischemic penumbra and core are differentially affected following transient focal cerebral ischemia in rats. I Cereb Blood Flow Metab 2004, 24(3):343-349.
23. Beynon C, Kiening KL, Orakcioglu B, Unterberg AW, Sakowitz OW: Brain tissue oxygen monitoring and hyperoxic treatment in patients with traumatic brain injury. J Neurotrauma 2012, 29(12):2109-2123.

24. Sun $L$, Strelow $H$, Mies $G$, Veltkamp R: Oxygen therapy improves energy metabolism in focal cerebral ischemia. Brain Res 2011, 1415:103-108.

25. Fisher M, Bastan B: Identifying and utilizing the ischemic penumbra. Neurology 2012, 79 (13 Suppl 1):S79-S85

26. Liu C, Weaver J, Liu KJ: Rapid conditioning with oxygen oscillation: neuroprotection by intermittent normobaric hyperoxia after transient focal cerebral ischemia in rats. Stroke 2012, 43(1):220-226.

27. Gao Z, Spilk S, Momen A, Muller MD, Leuenberger UA, Sinoway LI: Vitamin $C$ prevents hyperoxia-mediated coronary vasoconstriction and impairment of myocardial function in healthy subjects. Eur J Appl Physiol 2012, 112(2):483-492.

28. Shin HK, Dunn AK, Jones PB, Boas DA, Lo EH, Moskowitz MA, et al: Normobaric hyperoxia improves cerebral blood flow and oxygenation, and inhibits peri-infarct depolarizations in experimental focal ischaemia. Brain 2007, 130(Pt 6):1631-1642.

29. Ray PD, Huang BW, Tsuji Y: Reactive oxygen species (ROS) homeostasis and redox regulation in cellular signaling. Cell Signal 2012, 24(5):981-990

30. Allen $\mathrm{CL}$, Bayraktutan U: Oxidative stress and its role in the pathogenesis of ischaemic stroke. Int J Stroke 2009, 4(6):461-470.

31. Qi Z, Luo Y, Liu KJ: Free radicals and matrix metalloproteinases in bloodbrain barrier disruption after cerebral ischemia. Acta Biophys $\operatorname{Sin} 2012$, 28(5):383-391.

32. Agardh CD, Zhang H, Smith ML, Siesjo BK: Free radical production and ischemic brain damage: influence of postischemic oxygen tension. Int $J$ Dev Neurosci 1991, 9(2):127-138

33. Liu W, Sood R, Chen Q, Sakoglu U, Hendren J, Cetin O, et al: Normobaric hyperoxia inhibits NADPH oxidase-mediated matrix metalloproteinase- 9 induction in cerebral microvessels in experimental stroke. J Neurochem 2008, 107(5):1196-1205.

34. Singhal $A B$, Wang $X$, Sumii T, Mori $T$, Lo EH: Effects of normobaric hyperoxia in a rat model of focal cerebral ischemia-reperfusion. $J$ Cereb Blood Flow Metab 2002, 22(7):861-868.

35. Yuan Z, Liu W, Liu B, Schnell A, Liu KJ: Normobaric hyperoxia delays and attenuates early nitric oxide production in focal cerebral ischemic rats. Brain Res 2010, 1352:248-254

36. Liebner S, Czupalla CJ, Wolburg H: Current concepts of blood-brain barrier development. Int J Dev Biol 2011, 55(4-5):467-476.

37. Jian LK, Rosenberg GA: Matrix metalloproteinases and free radicals in cerebral ischemia. Free Radic Biol Med 2005, 39(1):71-80.

38. Jin X, Liu J, Yang Y, Liu KJ, Yang Y, Liu W: Spatiotemporal evolution of blood brain barrier damage and tissue infarction within the first $3 \mathrm{~h}$ after ischemia onset. Neurobiol Dis 2012, 48(3):309-316.

39. Liu J, Jin X, Liu KJ, Liu W: Matrix metalloproteinase-2-mediated occludin degradation and caveolin-1-mediated claudin-5 redistribution contribute to blood-brain barrier damage in early ischemic stroke stage. J Neurosci 2012, 32(9):3044-3057.

40. Rosell A, Ortega-Aznar A, Alvarez-Sabin J, Fernandez-Cadenas I, Ribo M, Molina CA, et al: Increased brain expression of matrix metalloproteinase9 after ischemic and hemorrhagic human stroke. Stroke 2006, 37(6):1399-1406.

41. Tejima E, Zhao BQ, Tsuji K, Rosell A, Van LK, Gonzalez RG, et al: Astrocytic induction of matrix metalloproteinase- 9 and edema in brain hemorrhage. J Cereb Blood Flow Metab 2007, 27(3):460-468.

42. Asahi M, Asahi K, Jung JC, del Zoppo GJ, Fini ME, Lo EH: Role for matrix metalloproteinase 9 after focal cerebral ischemia: effects of gene knockout and enzyme inhibition with BB-94. J Cereb Blood Flow Metab 2000, 20(12):1681-1689.

43. Ulrich NH, Dehmel T, Wittsack HJ, Kieseier BC, Seitz RJ: Peripheral blood levels of matrix metalloproteinase-9 predict lesion volume in acute stroke. Neurol Sci 2012, doi:10.1007/s10072-012-0999-8.

44. Copin JC, Bengualid DJ, Da Silva RF, Kargiotis O, Schaller K, Gasche Y: Recombinant tissue plasminogen activator induces blood-brain barrier breakdown by a matrix metalloproteinase-9-independent pathway after transient focal cerebral ischemia in mouse. Eur J Neurosci 2011, 34(7):1085-1092.

45. Geng X, Ren C, Wang T, Fu P, Luo Y, Liu X, et al: Effect of remote ischemic postconditioning on an intracerebral hemorrhage stroke model in rats. Neurol Res 2012, 34(2):143-148. 
46. Li Y, Kawamura S, Shirasawa M, Yasui N, Fukasawa H: Failure of normobaric oxygen therapy to reduce ischemic brain damage in rats. Undersea Hyperb Med 1994, 21(3):245-249.

47. Mickel HS, Kempski O, Feuerstein G, Parisi JE, Webster HD: Prominent white matter lesions develop in Mongolian gerbils treated with $100 \%$ normobaric oxygen after global brain ischemia. Acta Neuropathol 1990 79(5):465-472

48. Padma MV, Bhasin A, Bhatia R, Garg A, Singh MB, Tripathi M, et al: Normobaric oxygen therapy in acute ischemic stroke: a pilot study in Indian patients. Ann Indian Acad Neurol 2010, 13(4):284-288.

49. Fisher M, Feuerstein G, Howells DW, Hurn PD, Kent TA, Savitz SI, et al: Update of the stroke therapy academic industry roundtable preclinical recommendations. Stroke 2009, 40(6):2244-2250.

50. Stroke Therapy Academic Industry Roundtable (STAIR): Recommendations for standards regarding preclinical neuroprotective and restorative drug development. Stroke 1999, 30(12):2752-2758.

51. Hatcher MA, Starr JA: Role of tissue plasminogen activator in acute ischemic stroke. Ann Pharmacother 2011, 45(3):364-371.

52. Hiu T, Nakagawa S, Hayashi K, Kitagawa N, Tsutsumi K, Kawakubo J, et al: Tissue plasminogen activator enhances the hypoxia/reoxygenationinduced impairment of the blood-brain barrier in a primary culture of rat brain endothelial cells. Cell Mol Neurobiol 2008, 28(8):1139-1146.

53. Mishiro $K$, Ishiguro M, Suzuki $Y$, Tsuruma K, Shimazawa M, Hara H: A broadspectrum matrix metalloproteinase inhibitor prevents hemorrhagic complications induced by tissue plasminogen activator in mice. Neuroscience 2012, 205:39-48.

54. Sumii T, Lo EH: Involvement of matrix metalloproteinase in thrombolysisassociated hemorrhagic transformation after embolic focal ischemia in rats. Stroke 2002, 33(3):831-836.

55. Wang X, Tsuji K, Lee SR, Ning M, Furie KL, Buchan AM, et al: Mechanisms of hemorrhagic transformation after tissue plasminogen activator reperfusion therapy for ischemic stroke. Stroke 2004, 35(11 Suppl 1):2726-2730.

56. Wang X, Rosell A, Lo EH: Targeting extracellular matrix proteolysis for hemorrhagic complications of tPA stroke therapy. CNS Neurol Disord Drug Targets 2008, 7(3):235-242.

57. Rodriguez-Gonzalez R, Sobrino T, Rodriguez-Yanez M, Millan M, Brea D,

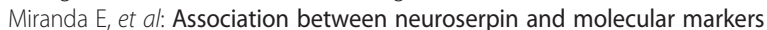
of brain damage in patients with acute ischemic stroke. J Trans/ Med 2011, 9:58.

58. Montaner J: Blood biomarkers to guide stroke thrombolysis. Front Biosci (Elite Ed) 2009, 1:200-208.

59. Henninger N, Fisher M: Normobaric hyperoxia - a promising approach to expand the time window for acute stroke treatment. Cerebrovasc Dis 2006, 21(1-2):134-136.

60. Henninger N, Bratane BT, Bastan B, Bouley J, Fisher M: Normobaric hyperoxia and delayed tPA treatment in a rat embolic stroke model. J Cereb Blood Flow Metab 2009, 29(1):119-129.

61. Kim HY, Singhal $A B$, Lo EH: Normobaric hyperoxia extends the reperfusion window in focal cerebral ischemia. Ann Neurol 2005, 57(4):571-575.

62. Fujiwara N, Murata Y, Arai K, Egi Y, Lu J, Wu O, et al: Combination therapy with normobaric oxygen (NBO) plus thrombolysis in experimental ischemic stroke. BMC Neurosci 2009, 10:79.

63. Jin X, Liu J, Liu KJ, Rosenberg GA, Yang Y, Liu W: Normobaric hyperoxia combined with minocycline provides greater neuroprotection than either alone in transient focal cerebral ischemia. Exp Neurol 2012, 240C:9-16.

64. Nonaka Y, Shimazawa M, Yoshimura S, Iwama T, Hara H: Combination effects of normobaric hyperoxia and edaravone on focal cerebral ischemia-induced neuronal damage in mice. Neurosci Lett 2008, 441(2):224-228.

65. Nonaka Y, Koumura A, Hyakkoku K, Shimazawa M, Yoshimura S, Iwama T, et al: Combination treatment with normobaric hyperoxia and cilostazol protects mice against focal cerebral ischemia-induced neuronal damage better than each treatment alone. J Pharmacol Exp Ther 2009, 330(1):13-22.

66. Topcuoglu MA, Saka E, Onal MZ: Hyperoxia potentiated sonothrombolysis as a method of acute ischemic stroke therapy. Med Hypotheses 2006, 66 (1):59-65.

67. Goldstein LB, Bushnell CD, Adams RJ, Appel LJ, Braun LT, Chaturvedi S, et al: Guidelines for the primary prevention of stroke: a guideline for healthcare professionals from the American Heart Association/American Stroke Association. Stroke 2011, 42(2):517-584
68. Adams HP, Del ZG, Alberts MJ, Bhatt DL, Brass L, Furlan A, et al: Guidelines for the early management of adults with ischemic stroke: a guideline from the American Heart Association/American Stroke Association Stroke Council, Clinical Cardiology Council, Cardiovascular Radiology and Intervention Council, and the Atherosclerotic Peripheral Vascular Disease and Quality of Care Outcomes in Research Interdisciplinary Working Groups: The American Academy of Neurology affirms the value of this guideline as an educational tool for neurologists. Circulation 2007, 115(20):e478-e534

doi:10.1186/2045-9912-3-2

Cite this article as: Qi et al:: Normobaric hyperoxia-based neuroprotective therapies in ischemic stroke. Medical Gas Research 2013 $3: 2$.

\section{Submit your next manuscript to BioMed Central and take full advantage of:}

- Convenient online submission

- Thorough peer review

- No space constraints or color figure charges

- Immediate publication on acceptance

- Inclusion in PubMed, CAS, Scopus and Google Scholar

- Research which is freely available for redistribution

Submit your manuscript at www.biomedcentral.com/submit
C) Biomed Central 